\title{
Sequential tunneling in doped superlattices: Fingerprints of impurity bands and photon-assisted tunneling
}

Wacker, Andreas; Jauho, Antti-Pekka; Zeuner, S.; Allen, S. K.

Published in:

Physical Review B Condensed Matter

Link to article, DOI:

10.1103/PhysRevB.56.13268

Publication date:

1997

Document Version

Publisher's PDF, also known as Version of record

Link back to DTU Orbit

Citation (APA):

Wacker, A., Jauho, A-P., Zeuner, S., \& Allen, S. K. (1997). Sequential tunneling in doped superlattices:

Fingerprints of impurity bands and photon-assisted tunneling. Physical Review B Condensed Matter, 56(20), 13268-13278. https://doi.org/10.1103/PhysRevB.56.13268

\section{General rights}

Copyright and moral rights for the publications made accessible in the public portal are retained by the authors and/or other copyright owners and it is a condition of accessing publications that users recognise and abide by the legal requirements associated with these rights.

- Users may download and print one copy of any publication from the public portal for the purpose of private study or research.

- You may not further distribute the material or use it for any profit-making activity or commercial gain

- You may freely distribute the URL identifying the publication in the public portal 


\title{
Sequential tunneling in doped superlattices: Fingerprints of impurity bands and photon-assisted tunneling
}

\author{
Andreas Wacker and Antti-Pekka Jauho \\ Mikroelektronik Centret, Danmarks Tekniske Universitet, DK-2800 Lyngby, Denmark \\ Stefan Zeuner* and S. James Allen \\ Center for Terahertz Science and Technology, University of California at Santa Barbara, Santa Barbara, California 93106
}

(Received 12 May 1997; revised manuscript received 28 August 1997)

\begin{abstract}
We report a combined theoretical and experimental study of electrical transport in weakly coupled doped superlattices. Our calculations exhibit negative differential conductivity at sufficiently high electric fields for all dopings. In low-doped samples the presence of impurity bands modifies the current-voltage characteristics substantially, and we find two different current peaks whose relative height changes with the electron temperature. These findings can explain the observation of different peaks in the current-voltage characteristics with and without external THz irradiation in low-doped samples. From our microscopic transport model we obtain quantitative agreement with the experimental current-voltage characteristics without using any fitting parameters. Both our experimental data and our theory show that absolute negative conductance persists over a wide range of frequencies of the free-electron laser source. [S0163-1829(97)07944-7]
\end{abstract}

\section{INTRODUCTION}

Perpendicular charge transport in biased superlattices is dominated by resonances due to the alignment of energy levels in different wells. These resonances yield distinct peaks in the current-voltage characteristics ${ }^{1,2}$ associated with negative differential conductivity (NDC) at fields above the peak. The instability associated with NDC causes the formation of electric-field domains ${ }^{3}$ as well as self-sustained oscillations in such structures. ${ }^{4}$ While for strongly coupled superlattices the electronic minibands dominate the electrical transport, ${ }^{5}$ in weakly coupled superlattices the transport is due to sequential tunneling from one well to the next. (For a discussion of the appropriate regimes see Refs. 6 and 7.) This situation was already regarded in Ref. 8 for tunneling between the lowest level and excited levels, in the adjacent well. There the current is driven by the different occupation of the two levels, and a maximum of the current occurs when the different levels are aligned. Tunneling between equivalent levels at low fields is slightly more complicated, as alignment occurs at zero field, where, of course, the current vanishes. The key point is the treatment of broadening of the states due to scattering which essentially determines the transport. This idea has been exploited to determine scattering rates by studying the transport between two quantum wells. $^{9-11}$ In the experiments, ${ }^{10,11}$ impurity scattering was diminished by the use of remote doping, which enabled one to study electron-electron scattering rates. In contrast to this, we focus on doped superlattices in the present paper. There impurity scattering at the ionized donors is an important scattering process whose impact we will examine in the following. In a previous study, ${ }^{12}$ a heavily doped sample was investigated, and good agreement with experimental data was found. Here we perform a systematic study of the lowfield transport in such structures for different doping densities. We find that the formation of impurity bands ${ }^{13,14}$ for low-doped samples causes a strong temperature dependence of the current-field relation which may display a double-peak structure at low fields.

If the superlattice is subjected to an external microwave field, photon-assisted tunneling (PAT) is possible where replica of the resonances are observed at biases which differ from alignment conditions ${ }^{15-17}$ by integer multiples of the photon energy. For certain field strengths of the irradiation field, absolute negative conductance has been observed experimentally. ${ }^{18,19}$ The main features of these experiments could be described qualitatively ${ }^{18-20}$ within the standard theory of photon-assisted tunneling ${ }^{21,22}$ but modifications due to photon sidebands from a single quantum well ${ }^{23}$ have also been suggested to explain the experimental findings. Here we present additional experimental data, and show that full quantitative agreement between theory and experiment can be found by a combination of a microscopic transport model with the standard theory of photon-assisted tunneling. This comparison strongly supports our claim that a microscopic treatment of impurity scattering is necessary for a full understanding of transport in low-doped superlattices.

The paper is organized as follows: Our transport model is presented in Sec. II. In order to understand the generic behavior, we give a phenomenological approximation, where many features can be seen analytically, in Sec. III. The calculated results for different doping densities are presented in Secs. IV and V using different screening models, respectively. Our calculations are compared with two different experiments concerning a highly doped and a low-doped sample in Sec. VI. In Sec. VII we consider transport under external irradiation. Finally, we will discuss the general significance of our results.

\section{MODEL}

We consider weakly coupled semiconductor quantum wells of period $d$. Then the electrons are essentially localized in the wells, and a reasonable basis set of wave functions is 
given by a product of Wannier functions $\Psi^{\nu}(z-j d)$, which are maximally localized ${ }^{24}$ in well $j$, and plane waves $e^{i \mathbf{k} \cdot \mathbf{r}}$. The $z$ direction is defined to be the growth direction and $\mathbf{k}$ and $\mathbf{r}$ are vectors within the $(x, y)$ plane. $\nu$ denotes the subband within the well. Here we restrict ourselves to the lowest level and omit the index $\nu$ in the following, and the energy of the lowest level is used as a reference point.

Regarding only next-neighbor coupling $T_{1}$ we have the following Hamiltonian ( $F$ is the electric field, and $e<0$ is the charge of the electron):

$$
\begin{aligned}
\hat{H}= & \sum_{j, \mathbf{k}}\left[\left(E_{k}-j e F d\right) a_{j}^{\dagger}(\mathbf{k}) a_{j}(\mathbf{k})+T_{1} a_{j+1}^{\dagger}(\mathbf{k}) a_{j}(\mathbf{k})\right. \\
& \left.+T_{1} a_{j}^{\dagger}(\mathbf{k}) a_{j+1}(\mathbf{k})\right]+\hat{H}^{\text {scatt }}
\end{aligned}
$$

with the in-plane kinetic energy $E_{k}=\hbar^{2} k^{2} /\left(2 m_{w}\right)$, where $m_{w}$ is the effective mass in the well. $a_{j}$ and $a_{j}^{\dagger}$ are the annihilation and creation operators of electrons in well $j$, respectively. $\hat{H}^{\text {scatt }}$ denotes the contribution due to scattering which is not $\mathbf{k}$-conserving.

Within the lowest order in the coupling $T_{1}$ the current density from the lowest level in well $j$ to the lowest level in well $j+1$ is given by ${ }^{25}$

$$
\begin{aligned}
J_{j \rightarrow j+1}= & \frac{2 e}{A} \sum_{\mathbf{k}}\left|T_{1}\right|^{2} \int_{-\infty}^{\infty} \frac{d E}{2 \pi \hbar} A_{j+1}(\mathbf{k}, E+e F d) A_{j}(\mathbf{k}, E) \\
& \times\left[n_{F}\left(E-\mu_{j}\right)-n_{F}\left(E+e F d-\mu_{j+1}\right)\right] .
\end{aligned}
$$

Here $e$ is the electron charge, $A$ is the sample area, and $\mu_{j}$ is the local chemical potential in well $j$ measured with respect to the energy of the lowest level. $n_{F}(\mathcal{E})=1 /[1+\exp (\mathcal{E} /$ $\left.k_{B} T_{e}\right)$ ], and $T_{e}$ is the electron temperature. $F d$ denotes the voltage drop per period $d$. The spectral function $A(\mathbf{k}, E)$ is calculated for a given intrawell scattering $\hat{H}^{\text {scatt }}$ via the retarded self-energy $\Sigma^{\text {ret }}(\mathbf{k}, E)$ :

$$
A(\mathbf{k}, E)=\frac{-2 \operatorname{Im}\left\{\Sigma^{\mathrm{ret}}(\mathbf{k}, E)\right\}}{\left(E-E_{k}-\operatorname{Re}\left\{\Sigma^{\mathrm{ret}}\right\}\right)^{2}+\left(\operatorname{Im}\left\{\Sigma^{\mathrm{ret}}\right\}\right)^{2}} .
$$

$\mu_{j}$ is related to the electron density $n_{j}$ in well $j$, via the relation

$$
n_{j}=\int_{-\infty}^{\infty} d E \rho_{j}(E) n_{F}\left(E-\mu_{j}\right)
$$

with the density of states

$$
\rho_{j}(E)=\frac{2}{2 \pi A} \sum_{\mathbf{k}} A_{j}(\mathbf{k}, E)
$$

where the factor 2 reflects the spin degeneracy.

In our microscopic calculation we proceed as follows: First we determine the coupling $T_{1}$ as well as the Wannier functions $\Psi(z-j d)$ for the given superlattice parameters (see Appendix A). Then we calculate the self-energy $\Sigma^{\text {ret }}(\mathbf{k}, E)$ for impurity scattering using the self-consistent single-site approximation shown in Fig. 1. The respective formulas are given in Appendix B. The matrix element for impurity scattering is calculated from the Coulomb potential of the individual ionized donors. Screening is treated in two

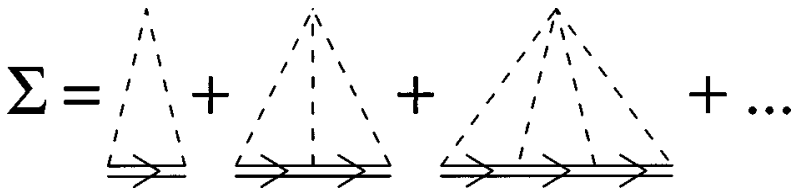

FIG. 1. The self-consistent single-site approximation. The dashed lines indicate impurity potentials and the double lines denote the full Green function.

different approaches, the random-phase approximation (RPA) for a free-electron gas and the Thomas-Fermi approximation (TF) using the actual density of states at the Fermi level (see Appendix C). Using the calculated spectral functions $A(\mathbf{k}, E)$, the chemical potential is determined by setting $n_{j}=N_{D}$ in Eq. (4), where $N_{D}$ is the doping density per period. Finally the current is calculated from Eq. (2). Note that all quantities used in the calculation are defined by the sample parameters and no fitting parameters are used.

\section{PHENOMENOLOGICAL DESCRIPTION}

In this section we want to provide some insight into the question how scattering affects the transport. Using a constant self-energy we derive some simple expressions for the current-field relation which will help to understand the full calculations presented in subsequent sections.

As mentioned in Sec. I, the level broadening essentially determines the transport in the sequential limit. This can be easily seen in the limit of vanishing scattering. Then the spectral functions become $\delta$-functions, $A(\mathbf{k}, E)$ $=2 \pi \delta\left(E-E_{k}\right)$. In this case the current vanishes for $e F d$ $\neq 0$. (In addition, further resonances may occur at finite fields, when the lowest level is aligned with higher levels in the neighboring well, which are not considered here.) Assuming $e F d \geqslant 0$, we rewrite Eq. (2) as follows:

$$
\begin{aligned}
J_{j \rightarrow j+1}= & e \frac{T_{1}^{2}}{\hbar} \int_{-\infty}^{\infty} d E\left\langle A_{j+1}\right\rangle(E, F) \rho_{j}(E)\left[n_{F}\left(E-\mu_{j}\right)\right. \\
& \left.-n_{F}\left(E+e F d-\mu_{j+1}\right)\right],
\end{aligned}
$$

with

$$
\left\langle A_{j+1}\right\rangle(E, F)=\frac{\int_{0}^{\infty} d E_{k} A_{j}(\mathbf{k}, E) A_{j+1}(\mathbf{k}, E+e F d)}{\int_{0}^{\infty} d E_{k} A_{j}(\mathbf{k}, E)},
$$

where we used Eq. (5) and performed the continuum limit. Now let us assume a constant self-energy $\sum^{\text {ret }}(\mathbf{k}, E)=-i \Gamma / 2$ in Eq. (7) for the sake of simplicity. Then the spectral functions become Lorentzians $A(\mathbf{k}, E)=\Gamma /\left[\left(E-E_{k}\right)^{2}+\Gamma^{2} / 4\right]$. Extending the lower integration to $-\infty$, we obtain

$$
\left\langle A_{j+1}\right\rangle=\frac{2 \Gamma}{(e F d)^{2}+\Gamma^{2}},
$$

which only depends on $F$. Note that this simple model with a constant self-energy cannot be used in the calculation of the density of states (5) as the integral for the electron density (4) diverges in this case. Therefore we use the freeelectron density of states $\rho_{j}(E)=\rho_{0} \Theta(E)$ [with $\left.\rho_{0}=m /\left(\pi \hbar^{2}\right)\right]$, and obtain, for equal chemical potentials $\mu_{j}=\mu_{j+1}=\mu$, 


$$
J(F)=e \rho_{0} \frac{T_{1}^{2}}{\hbar} \frac{2 \Gamma}{(e F d)^{2}+\Gamma^{2}} \int_{0}^{e F d} d E n_{F}(E-\mu) .
$$

For low electron temperature and voltage drop $\left(k_{B} T_{e}, e F d \ll \mu\right)$, we find

$$
J(F)=e \rho_{0} \frac{T_{1}^{2}}{\hbar} \frac{2 \Gamma e F d}{(e F d)^{2}+\Gamma^{2}} .
$$

Thus, we recover an Ohmic behavior for low fields $e F d \ll \Gamma$, a maximum of $J(F)$ at $e F d=\Gamma$, and negative differential conductivity for $e F d>\Gamma$. Equation (10) has been essentially used in Refs. 10 and 11 for the determination of scattering rates $\Gamma / \hbar$ from tunneling between two two-dimensional electron gases. Similar models using a phenomenological broadening $\Gamma$ were applied to sequential tunneling in superlattices in Refs. 20 and 26. The current at the maximum is given by

$$
J_{\max }=J\left(\frac{\Gamma}{e d}\right)=e \rho_{0} \frac{T_{1}^{2}}{\hbar},
$$

which is independent of doping, scattering, and temperature in the limit of $\mu \gg e F d, k_{B} T_{e}$ considered here.

If $k_{B} T_{e}$ becomes of the order of $\mu$ the factor $\int_{0}^{e F d} d E n_{F}(E-\mu)$ in Eq. (9) is smaller than $e F d$, and we obtain a decrease of the current with temperature. Here we have to take into account the temperature dependence of the chemical potential $\mu$. From Eq. (4) we find $1+\exp \left(\mu / k_{B} T_{e}\right)=\exp \left(n / \rho_{0} k_{B} T_{e}\right)$. This gives a zero-field conductivity

$$
\frac{d J}{d F}_{\mid F=0}=\frac{2 e^{2} \rho_{0}}{\hbar} \frac{d T_{1}^{2}}{\Gamma}\left[1-\exp \left(-\frac{n}{\rho_{0} k_{B} T_{e}}\right)\right],
$$

which is almost constant for $k_{B} T_{e}<n / \rho_{0}$ and drops as $1 / k_{B} T_{e}$ for large temperatures as observed experimentally in Ref. 27. For completeness, we give the result in the hightemperature limit $\left(k_{B} T_{e} \gg e F d, n / \rho_{0}\right)$

$$
J(F)=\frac{e n}{k_{B} T_{e}} \frac{T_{1}^{2}}{\hbar} \frac{2 \Gamma e F d}{(e F d)^{2}+\Gamma^{2}},
$$

which follows directly from Eq. (9). It is interesting to note that Eq. (10) is identical to the current-field relation calculated for miniband conduction ${ }^{28}$ using a constant scattering time $\hbar / \Gamma$ for $\mu>2 T_{1}$ and $k_{B} T_{e}=0$. Equivalently, Eq. (13) was obtained from miniband transport in the limit $k_{B} T_{e} \gg 2 T_{1}, n / \rho_{0}$ as well. ${ }^{29}$ This shows that the models of sequential tunneling and miniband conduction give the same results provided either the electron temperature or the electron density are large.

\section{RESULTS FOR RPA SCREENING}

As a model system we choose an $\mathrm{Al}_{0.3} \mathrm{Ga}_{0.7} \mathrm{As}-\mathrm{GaAs}$ superlattice with barrier width $b=10 \mathrm{~nm}$ and well width $w=10$ $\mathrm{nm}$. We use the conduction-band offset $240 \mathrm{meV}$, and the effective masses $m_{w}=0.067 m_{e}$ and $m_{b}=0.0919 m_{e}$ (Ref. 30) in the Kronig-Penney model, yielding a coupling $T_{1}=-0.0116 \mathrm{meV}$. We assume $\delta$ doping in the middle of the quantum wells. The interaction with impurities located in

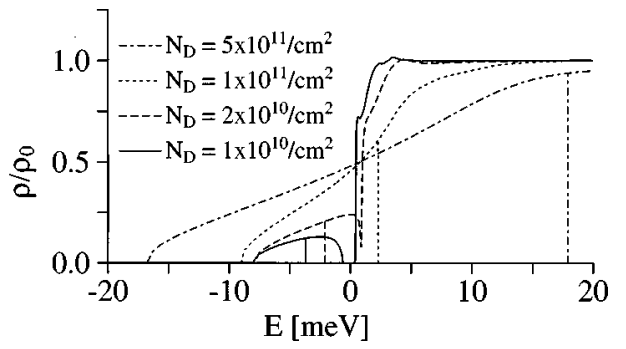

FIG. 2. Calculated density of states in units of the 2D freecarrier density $\rho_{0}$ using RPA screening. The vertical lines indicate the position of the chemical potential for $T=0$ at the respective doping densities.

different wells is found to be negligibly small. In this section screening is treated within the RPA, assuming a free-electron gas.

\section{A. Density of states}

In Fig. 2 we show the resulting densities of states for four different doping densities $N_{D}$. For high $N_{D}$ the density of states exhibits a monotonic increase from $\rho=0$ at $E \leqslant E_{\min }$ to $\rho \sim \rho_{0}$ for $E \rightarrow \infty$, where $E_{\min }$ denotes the lowest edge of the density of states. In contrast to this, the density of states splits into two parts for small doping: $\rho(E)$ takes finite values in a certain region below $E=0$, which we will refer to as an impurity band. For higher energies $\rho(E)$ is quite similar to the density of states of the free-electron gas. These results are in good agreement with the findings of Ref. 14. The onset of the impurity band occurs at slightly larger energies $E_{\text {min }}$ here, as the wave functions are less confined due to the spreading into the barrier which was neglected in Ref. 14.

We also marked the positions of the Fermi level $E^{F}$ (i.e., the chemical potential for $T_{e}=0$ ). For low densities the position is just in the middle of the impurity band, indicating that the impurity band consists of exactly two states per impurity due to the assumed spin degeneracy. (This degeneracy would be lifted if spin-resolved interaction was taken in account; also see Appendix B.) For high densities the position of $E^{F}$ roughly equals the Fermi level of the free-electron gas $N_{D} / \rho_{0}$. The crossover between these two limits occurs at $N_{D} \approx 5 \times 10^{10} / \mathrm{cm}^{2}$, where $E^{F} \approx 0$.

The respective spectral functions are plotted in Fig. 3. For $E=5 \mathrm{meV} A(k, E)$ resembles a Lorentzian centered close to $E_{k} \approx E$. This is the generic behavior of a free quasiparticle with a finite lifetime due to scattering. The width of the spectral functions is increasing with doping due to the enhanced scattering. We find a full width at half maximum $\Gamma=0.5 \mathrm{meV}$ for $N_{D} \approx 1 \times 10^{10} / \mathrm{cm}^{2}$ and $\Gamma=5 \mathrm{meV}$ for $N_{D} \approx 1 \times 10^{11} / \mathrm{cm}^{2}$, which are in the range of the calculated values of $-2 \operatorname{Im}\{\Sigma(\mathbf{k}, E)\}$.

For $E=-5 \mathrm{meV}$ the spectral functions exhibit a monotonic decrease. For high doping the slope is comparable to the slope at $E=5 \mathrm{meV}$. In contrast to this, the spectral function for $E=5$ and $-5 \mathrm{meV}$ are entirely different for low doping, indicating that two different types of states occur. While the states for $N_{D}=10^{10} / \mathrm{cm}^{2}$ are essentially freeparticle states at $E=5 \mathrm{meV}$, they are localized in space for $E=-5 \mathrm{meV}$, which is the signature of an impurity band. ${ }^{13}$ 

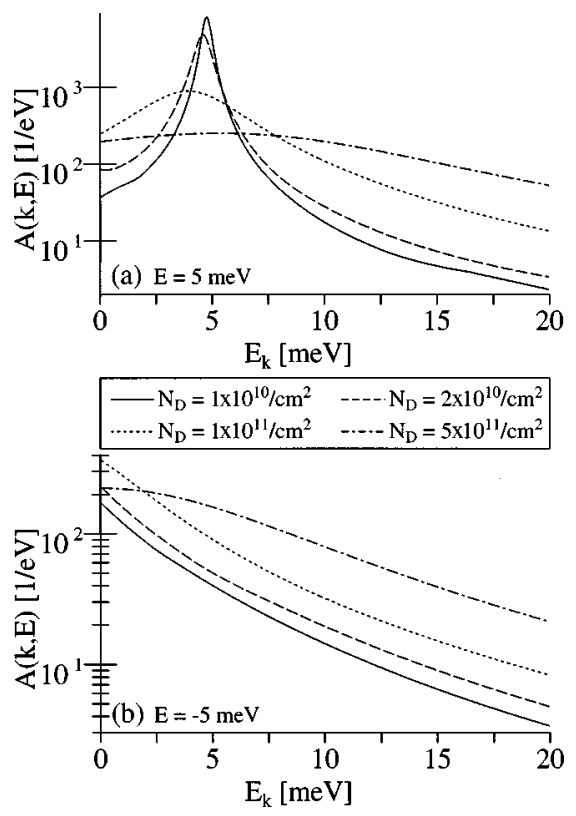

FIG. 3. Calculated spectral functions $A(E, k)$ vs $E_{k}=\hbar^{2} k^{2} / 2 m$ using RPA screening for different doping densities at $E=5 \mathrm{meV}$ (a) and $5 \mathrm{meV}(\mathrm{b})$.

\section{B. Currents}

We calculate the current densities $J_{j \rightarrow j+1}(e F d)$ for different electron temperatures $T_{e}$ from Eq. (2). The results are shown in Fig. 4. For all temperatures and densities we find an Ohmic range for low electric fields and negative differential conductance for high electric fields. Let us first regard the high doping case [Figs. 4(a)-4(c)], where no impurity bands form, and where the Fermi level is significantly above $E=0$. In this case approximation (9) is justified, and indeed we find a maximum at values of $e F d$ which are in the range of calculated values of $\Gamma=-2 \operatorname{Im}\left\{\sum^{\mathrm{ret}}(\mathbf{k}, E)\right\}$. The height is
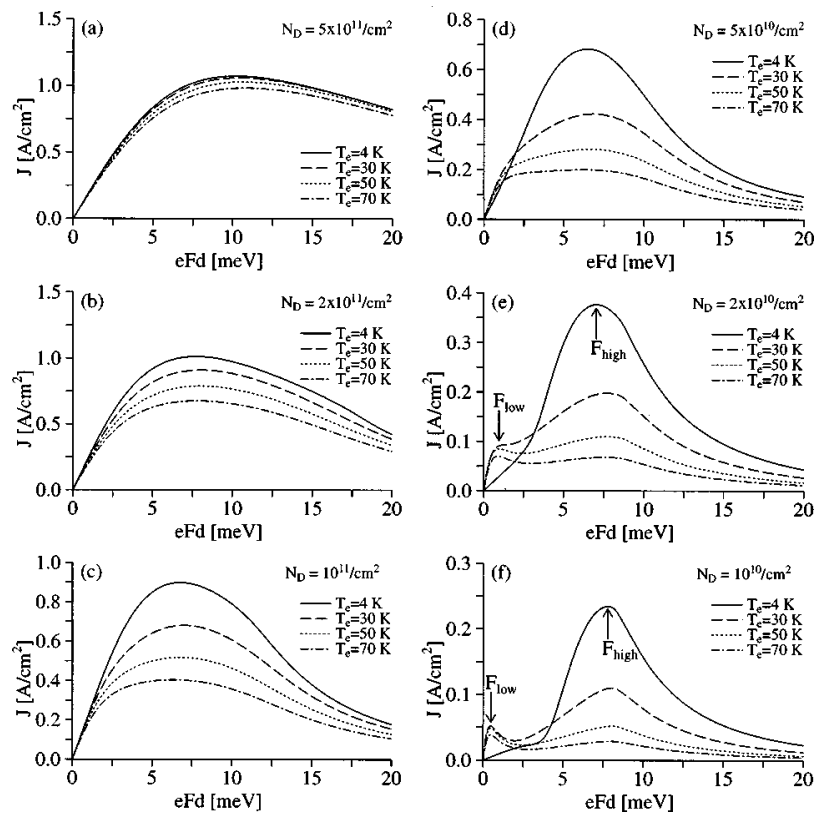

FIG. 4. Calculated temperature dependence of the current-field relations for different doping densities. The screening is treated within the RPA.

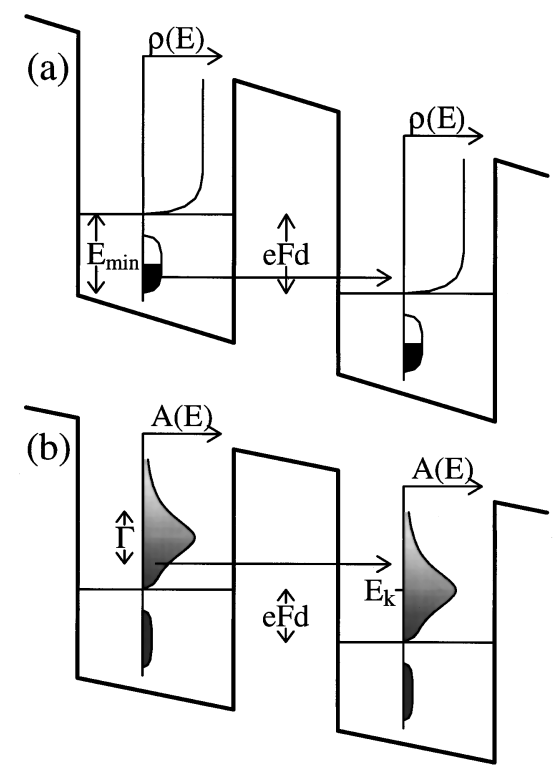

FIG. 5. Explanation of the two different current maxima within a sketch of the conduction-band profile: (a) For low temperatures the electrons occupy the impurity band (black area). As these states exhibit a flat spectral function [see Fig. 3(b)], they contain contributions from essentially all $\mathbf{k}$ vectors and thus tunneling into the free-particle states is possible at all energies. Maximal current is found when all states from the impurity band can tunnel into the free-particle states, i.e., $e F d \approx\left|E_{\min }\right|$. (b) For high temperatures, the electrons occupy the free-electron states as well (the grey scale indicates the occupation given by the Fermi-function). The spectral function $A(\mathbf{k}, E)$ of such a free-electron state with given wave vector $\mathbf{k}$ is peaked around $E=E_{k}$ as shown in the figure. Due to $\mathbf{k}$ conservation, tunneling can only take place if the spectral functions for the same $\mathbf{k}$ of both wells overlap. On the other hand, a net current is caused by the difference in occupation. This competition results in a current maximum for $e F d \approx \Gamma$ as shown in Sec. III.

estimated by $J_{\max }=0.91 \mathrm{~A} / \mathrm{cm}^{2}$ from Eq. (11), which is in good agreement with the full calculation at $T_{e}=4 \mathrm{~K}$. Note that the maximal current is almost independent on the doping in this range. For $N_{D}=5 \times 10^{11} / \mathrm{cm}^{2}$ the chemical potential is larger than $k_{B} T_{e}$ for all temperatures. Thus the current is hardly affected by the temperature. In contrast to this, the current drops with temperature for lower doping $\left(N_{D}=10^{11} / \mathrm{cm}^{2}\right)$. All these findings are in good agreement with the phenomenological description using a constant $\Gamma$ discussed above.

For low-doped samples [see Figs. 4(e) and 4(f)], another scenario occurs. Here we find two different maxima in the current-field relation whose relative weight is changed by temperature. The reason for this behavior is the presence of impurity bands for these doping levels. For $T_{e}=4 \mathrm{~K}$ we find a maximum at $e F_{\text {high }} d \approx 8 \mathrm{meV}$. This is due to tunneling from the impurity band to the free states [see Fig. 5(a)]. The maximum occurs at the energy where the bottom of the impurity band in one well is aligned with the band edge of the free-electron states in the neighboring well, i.e., $e F_{\text {high }} d$ $\approx\left|E_{\text {min }}\right|$. An increasing temperature leads to a transfer of electrons from the impurity band to the free-electron states, and consequently the current at $e F_{\text {high }} d$ decreases with increasing $T_{e}$. The density of states in the impurity band is much lower than in the free-electron states, and hence the 


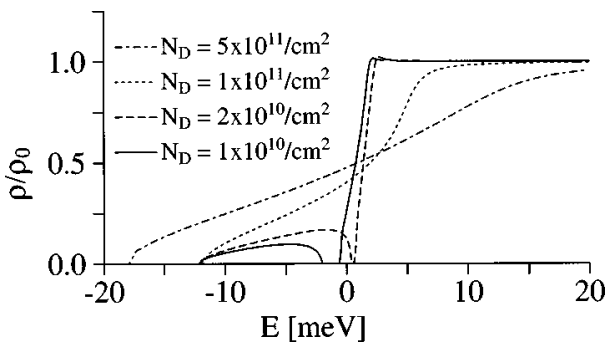

FIG. 6. Calculated density of states in units of the 2D free carrier density $\rho_{0}$ using Thomas-Fermi screening for different doping densities.

majority of the electrons will be in the free-electron states for $k_{B} T_{e} \gtrsim\left|E_{\text {min }}\right|$ [see Fig. 5(b), where the grey scale denotes the relative occupation]. The current contribution due to the freeelectron states can be understood within the phenomenological constant- $\Gamma$ approach. There is a maximum at $e F_{\text {low }} d \approx \Gamma$, which coincides with the full width at half maximum of the spectral function at $E=5 \mathrm{meV}$ in Fig. 3. The amplitude of this maximum depends on two competing effects: On the one hand, the occupation of the free-electron states increases with temperature. On the other hand, the Fermi factor in Eq. (9) strongly decreases with temperature. This explains the calculated behavior, where the peak at $e F_{\text {low }} d$ takes its maximum at intermediate temperatures.

\section{RESULTS FOR THOMAS-FERMI SCREENING}

The properties related with the formation of impurity bands are sensitive to the actual screening of the interaction. ${ }^{13}$ For low doping densities the density of states differs essentially from the free-electron density of states and thus the use of RPA screening by a free-electron gas is questionable. In order to take this effect into account we use the $\mathrm{TF}$ approximation with the actual density of states at the Fermi level (see Appendix C) in this section. Of course neither the free-electron RPA nor the TF approximation treat the screening entirely correctly, but we hope to obtain some insight into the general features by comparing these two approaches.

In Fig. 6 we show the resulting density of states which is in qualitative agreement with the results of the RPA screening (Fig. 2). For $N_{D}=5 \times 10^{11} / \mathrm{cm}^{2}$, the density of states is almost identical, while for lower densities some deviations occur. Especially the onset of the impurity band $E_{\min }$ is shifted to lower energies for TF screening. Furthermore the impurity bands extend over a larger energy range and have a lower density of states, so that the total density is conserved. The reason for these deviations lies in the fact that TF screening is less effective than RPA screening if the actual density of states at the Fermi level is used. Therefore both the binding energy of the impurities as well as the broadening of the states become larger.

This manifests itself in the calculated current densities (see Fig. 7). For high doping (a), the characteristics are almost identical, while for lower doping deviations occur. At first, note that the maxima due to tunneling between freeelectron states (the maximum for $N_{D}=10^{11} / \mathrm{cm}^{2}$ as well as the maxima $F_{\text {low }}$ for $N_{D}=2 \times 10^{10} / \mathrm{cm}^{2}$ and $N_{D}=10^{10} / \mathrm{cm}^{2}$ ) are shifted to the right according to the stronger scattering,
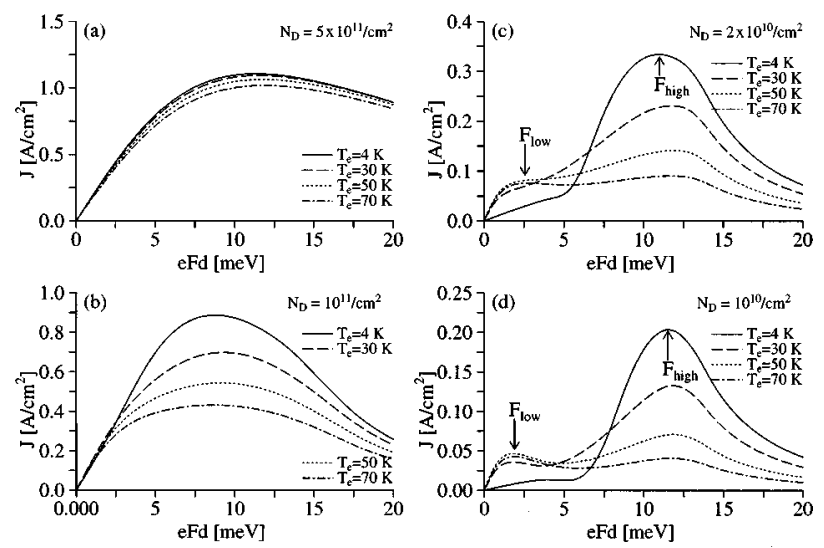

FIG. 7. Calculated temperature dependence of the current-field relations for different doping densities using $\mathrm{TF}$ screening.

which increases $\Gamma$. Second the peak at $F_{\text {high }}$ is shifted to the right compared to Fig. 4. Again we find $e F_{\text {high }} d \approx E_{\min }$ for both densities.

Therefore we conclude that within both approximations for screening the two maxima are determined by specific quantities describing the scattering. $e F_{\text {low }} d$ reflects the average broadening $\Gamma$ of the free-particle states, and $e F_{\text {high }} d$ is the energy separation between the onset of the impurity band and the free-particle states.

\section{COMPARISON WITH EXPERIMENTS}

Previously, ${ }^{12}$ the formalism was applied for the highly doped sample $\left(N_{D}=8.75 \times 10^{11} / \mathrm{cm}^{2}\right)$ of Refs. 31 and 32 . Good quantitative agreement with the experimental data was found, albeit using a barrier width being $10 \%$ smaller than the nominal value. (A similar width had been used in the original analysis by the experimentalists as well. ${ }^{32}$ ) The position of the first maximum occurred at $e F d=13 \mathrm{meV}$, which is almost independent of the barrier width (which mainly changes $T_{1}$ ) and in excellent agreement with the experimental finding. The second resonance, as well as the formation of field domains, was also studied in Ref. 12, and again good agreement with experimental data was found.

A low-doped superlattice $\left(N_{D}=6 \times 10^{9} / \mathrm{cm}^{2}, b=5 \mathrm{~nm}\right.$, $w=15 \mathrm{~nm}, A=8 \mu \mathrm{m}^{2}$ ), with $N=10$ wells, was used in the experiments of Refs. 18 and 19 in order to study the transport under strong $\mathrm{THz}$ irradiation from a free-electron laser. Additional data for this sample will be given in the following. Without irradiation a broad maximum was found in the range $50 \mathrm{mV}<U<100 \mathrm{mV}$, where the current is almost constant. For $U>100 \mathrm{mV}$, domain formation sets in. Dividing by the number of periods $(N=10)$, the maximum extends to $e F_{\text {unirr }} d \approx 10 \mathrm{meV}$. In contrast to this, the photon replica under strong $\mathrm{THz}$ irradiation could be consistently explained by assuming an "instantaneous" current-voltage characteristic $^{19}$ with a distinct maximum at $U \approx 20 \mathrm{mV}$ (i.e., $e F_{\text {irr }} d=2 \mathrm{meV}$ ).

We calculate the current-field relation for this superlattice using the experimental sample parameters. In order to model the homogeneous doping we use eight equally spaced $\delta$-doping layers per period. The calculated density of states for both RPA and TF screening is shown in Fig. 8. The 


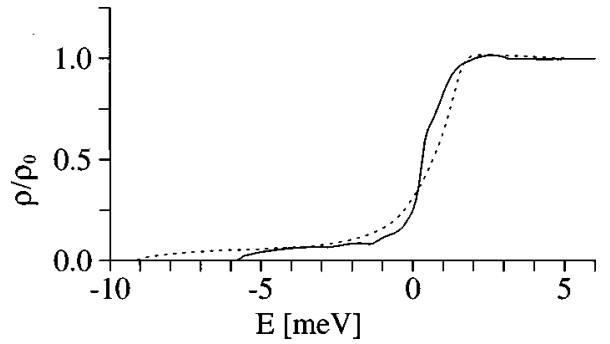

FIG. 8. Calculated density of states for the sample parameters of Refs. 18 and 19 using RPA screening (full line) and TF screening (dashed line).

density of states resembles the result for low doping found before. Nevertheless we do not find a separation between the impurity band and the free-particle states. The reason is the homogeneous doping: The different impurity positions have different binding energies which smear the impurity band. Again the onset of the impurity band occurs at significantly lower energies within the reduced Thomas-Fermi screening. Both values of $\left|E_{\text {min }}\right|$ are smaller than the corresponding values for low doping for the calculation done before (see Figs. 2 and 6). This is due to the larger well width in the sample: The Wannier states are less localized and therefore the matrix-element for impurity scattering (B2) as well as the binding energy of the impurity levels is smaller.

The results for the current-field relation are shown in Fig. 9. Again we find two maxima whose relative height changes with temperature. The position of the maximum for low temperatures, $e F_{\text {high }} d$, is almost identical to the value of $\left|E_{\min }\right|$ for both types of screening like in the calculations shown before.

Now we can offer an explanation for the two different maxima occurring in the experiment ${ }^{18,19}$ with and without irradiation mentioned above. For low electron temperatures and without irradiation, the maximum at $e F_{\text {high }} d$ dominates the transport, and thus domain formation sets in at voltages exceeding $U \approx N e F_{\text {high }} d$, where $N=10$ is the number of wells. If the $\mathrm{THz}$ radiation is present, the electrons are excited from the impurity band into the free-electron states corresponding to a larger effective electron temperature. Thus the maximum at $U=N e F_{\text {low }} d$ is dominant, and the photon replicas corresponding to this feature are seen experimentally. The experimental values therefore suggest $e F_{\text {high }} d=10$ $\mathrm{meV}$ and $e F_{\text {low }} d=2 \mathrm{meV}$, which is in excellent agreement with the calculation using Thomas-Fermi screening. This indicates that the RPA, using a free-electron gas, overestimates the screening in low-doped samples. The Thomas-Fermi approximation with the actual density of states at the Fermi level seems to reproduce the experimental results better in agreement with our argumentation in Appendix C. Therefore we will use it in the following for comparison with the experiment.

In Fig. 10 we compare the calculated currents with the experimental current-voltage characteristic without irradiation in a wider range of fields. Here we included the resonance around $e F d \approx 50 \mathrm{meV}$ between the lowest level and the first excited level as well. The calculation of the corresponding current is completely analogous to Eq. (2); for details, see Ref. 7. Note that there are no fitting parameters involved in the calculation - all quantities including matrix
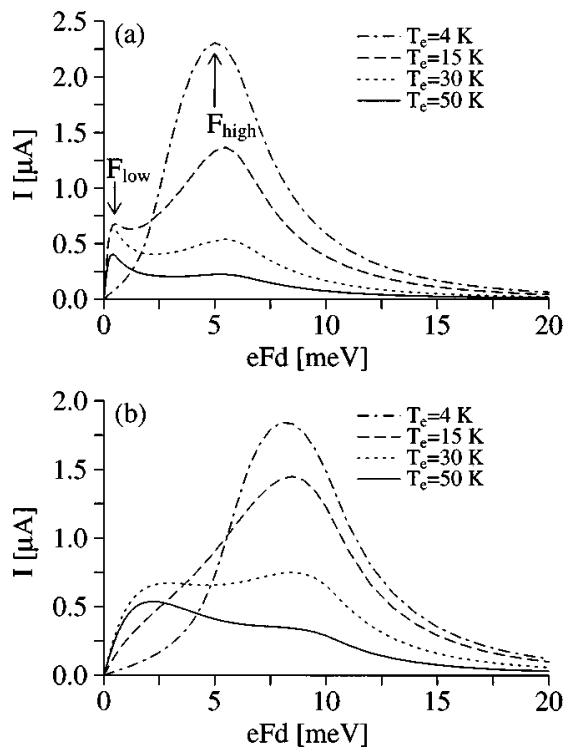

FIG. 9. Calculated temperature dependence of the current-field relations for the sample of Refs. 18 and 19. The screening is treated within the RPA (a) and within the Thomas-Fermi approximation (b).

elements and spectral functions are directly calculated for the given sample parameters as outlined above. Let us first focus on the low-field region. For $U<10 \mathrm{mV}$ the experimental data are in good agreement with the calculated currents for $T_{e}=4$ $\mathrm{K}$, the experimental lattice temperature. With increasing bias, the experimental data leave the 4-K curve and follow the $T_{e}=35 \mathrm{~K}$ curve at the plateau between 50 and $100 \mathrm{mV}$. This can be understood by electron heating inside the sample: For a voltage drop of $8 \mathrm{mV}$ per period and a current of $0.6 \mu \mathrm{A}$ the Joule heating is $P \approx 10 \mathrm{pW}$ per electron. In a recent transport experiment, a distribution function with $T_{e} \approx 40 \mathrm{~K}$ was observed ${ }^{33}$ for this amount of heating, albeit using a sample with higher doping. This shows that the electron temperature strongly deviates from the lattice temperature in the experiment considered in good agreement with our findings. At $U>100 \mathrm{mV}$, the homogeneous field distri-

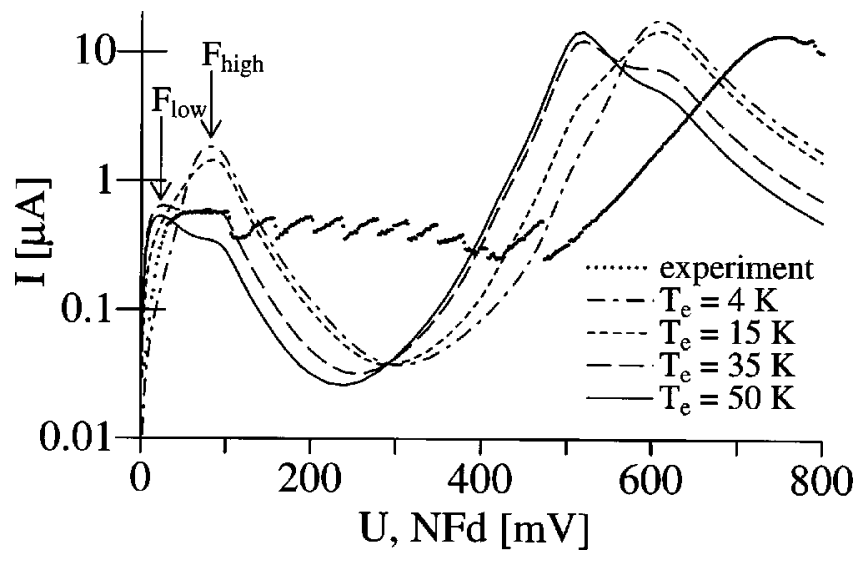

FIG. 10. Experimental current-voltage characteristic without external irradiation together with calculations for different electron temperatures. In the calculation we estimate the bias by $N F d$ assuming a homogeneous field distribution and neglecting potential drops in the contact regions. 


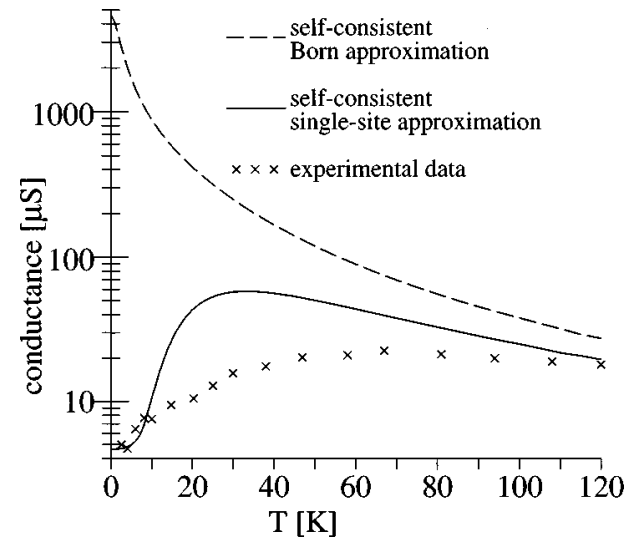

FIG. 11. Temperature dependence of the zero-bias conductance for the sample of Refs. 18 and 19. Full line: calculation using spectral functions from the single-site approximation and TF screening. Dashed line: calculation using spectral functions from the selfconsistent Born approximation. Crosses: experimental data.

bution becomes unstable, as the region of negative differential conductance is reached and electric-field domains form, causing the sawtooth shape of the characteristic (see Ref. 7, and references cited therein). For $U>450 \mathrm{mV}$ one can clearly see the resonance between the lower level and the first excited level in the well which is located $48 \mathrm{meV}$ above the ground level. Again the calculation exhibits two different peaks depending on the electron temperature due to the different occupation of the impurity bands, although only the high-temperature result should be meaningful due to the heating of the carriers. The peak height of around $14 \mu \mathrm{A}$ is in excellent agreement with the value of $13.6 \mu \mathrm{A}$ found experimentally for our sample. The experimental peak position is located at a higher bias. This may be due to a voltage drop in the receiving contact, where a low-doped spacer layer of $d_{\text {contact }}=50 \mathrm{~nm}$ thickness exists. If the electric field inside the sample is large, it cannot be screened within the spacer layer, and the effective field inside the sample is $U \sim\left(N d+d_{\text {contact }}\right) F$ instead of $U=N F d$ as assumed in the figure.

In order to circumvent the problems of electron heating, we investigated the temperature dependence of the zero-bias conductance $G=d I / d U$, where $T_{e}$ should be equal to the lattice temperature $T$. The results are shown in Fig. 11 both for our full calculation using TF screening as well as for spectral functions calculated within the self-consistent Born approximation (B7), where no impurity bands form. In the latter case $G$ is monotonously decreasing in $T$, as shown in Fig. 11. This can be easily understood within the phenomenological constant- $\Gamma$ approach, Eq. (12). However, a different scenario emerges if the electrons occupy impurity bands for low temperatures. Then $G$ is strongly suppressed due to the small values of $A(\mathbf{k}, E)$ for $E<0$; see Fig. 3. As temperature is increased, more electrons are excited to the freeelectron states, and $G$ increases with $T$ until the impurity bands are almost empty at $k_{B} T \sim\left|E_{\min }\right|$. This physical picture is confirmed by the experimental data shown in Fig. 11. At low temperatures, the agreement is quantitative, while at intermediate $T$ the theory overestimates $G$; this is most likely due to additional scattering processes not included in our calculation, or by the presence of a contact resistance which may limit the experimental conductance.

Thus we may conclude that the results of our calculations are in good agreement with experimental data both for high and low doping. Nevertheless, a direct observation of the two-peak structure is not available so far.

\section{PHOTON-ASSISTED TUNNELING}

The standard theory of PAT considers tunneling between two reservoirs between which a dc voltage $\Delta U_{\mathrm{dc}}$ is applied. Let us denote the current-voltage characteristic $(I-V)$ without irradiation by $I^{\mathrm{dc}}\left(\Delta U_{\mathrm{dc}}\right)$. Under irradiation an additional ac bias $U=\Delta U_{\mathrm{ac}} \cos (2 \pi \nu t)$ is induced between the reservoirs. Then the time-averaged $I-V$ is given by ${ }^{22}$

$$
I^{\mathrm{irr}}\left(\Delta U_{\mathrm{dc}}\right)=\sum_{l=-\infty}^{\infty}\left[J_{l}(\alpha)\right]^{2} I^{\mathrm{dc}}\left(\Delta U_{\mathrm{dc}}+\frac{l h \nu}{e}\right),
$$

where $\alpha=e \Delta U_{\mathrm{ac}} /(h \nu)$ and $J_{l}$ is the ordinary Bessel function of order $l$. Thus, the current under irradiation is given as a sum over the photon replicas $e \Delta U_{\mathrm{dc}}+l h \nu$, where the alignment of the wells is shifted by integer multiples of the photon energy. The great advantage of Eq. (14) is that it expresses all transport properties in terms of $I^{\mathrm{dc}}\left(\Delta U_{\mathrm{dc}}\right)$. Equation (14) has been applied to photon-assisted tunneling in weakly coupled superlattices by identifying $\Delta U_{\mathrm{dc}}=F_{\mathrm{dc}} d$ and $\Delta U_{\mathrm{ac}}=F_{\mathrm{ac}} d$, where $F_{\mathrm{ac}}$ is the field component of the microwave field in the growth direction of the superlattice. ${ }^{16,17,19}$ Note that Eq. (14) also holds within a miniband model for strongly coupled superlattices. ${ }^{34}$

In Refs. 18 and 20 the $I^{\mathrm{dc}}\left(e F_{\mathrm{dc}} d\right)$ curve was calculated phenomenologically, and a qualitative agreement with the experimental data could be obtained. To refine the theory we use the results of our microscopic calculation (see Fig. 10) here. As the external irradiation heats the electronic distribution for zero bias as well, we use the curve for $T_{e}=35 \mathrm{~K}$. At this electron temperature about $50 \%$ of the electrons are occupying the states in the impurity band. Of course the actual electron distribution may deviate from a Fermi distribution under the strong irradiation. Nevertheless we expect that an effective temperature approach gives a reasonable description of the excited carriers. Further calculations show that the results for $T_{e}=30$ or $40 \mathrm{~K}$ do not differ qualitatively. Quantitative agreement between theory [Fig. 12(a)] and experiment [Fig. 12(b)] is found for $h \nu=6.3 \mathrm{meV}(1.5 \mathrm{THz})$ for different strengths of the laser field. We find a direct tunneling peak at $U_{\mathrm{dir}}=N F_{\text {low }} d \approx 20 \mathrm{mV}$ and photon replicas at $U \approx U_{\mathrm{dir}}+N h \nu / e=83 \mathrm{mV}$ and $U \approx U_{\mathrm{dir}}+2 N h \nu / e=146 \mathrm{mV}$. Photon replicas of the second peak around $N F_{\text {high }} d \approx 100$ $\mathrm{mV}$ are less pronounced as this peak is broader. Our calculations show that they become visible if larger photon energies are used. For low bias and high intensities there is a region of absolute negative conductance, ${ }^{18}$ which we focus on in the following.

In Fig. 12(d) the laser intensity has been tuned such that maximal absolute negative conductance occurred for each of the different laser frequencies. Then we observe a minimum in the current at $U \approx-U_{\mathrm{dir}}+N h \nu / e$, which is just the first photon replica of the direct tunneling peak on the negativebias side. This replica dominates the current if the direct 

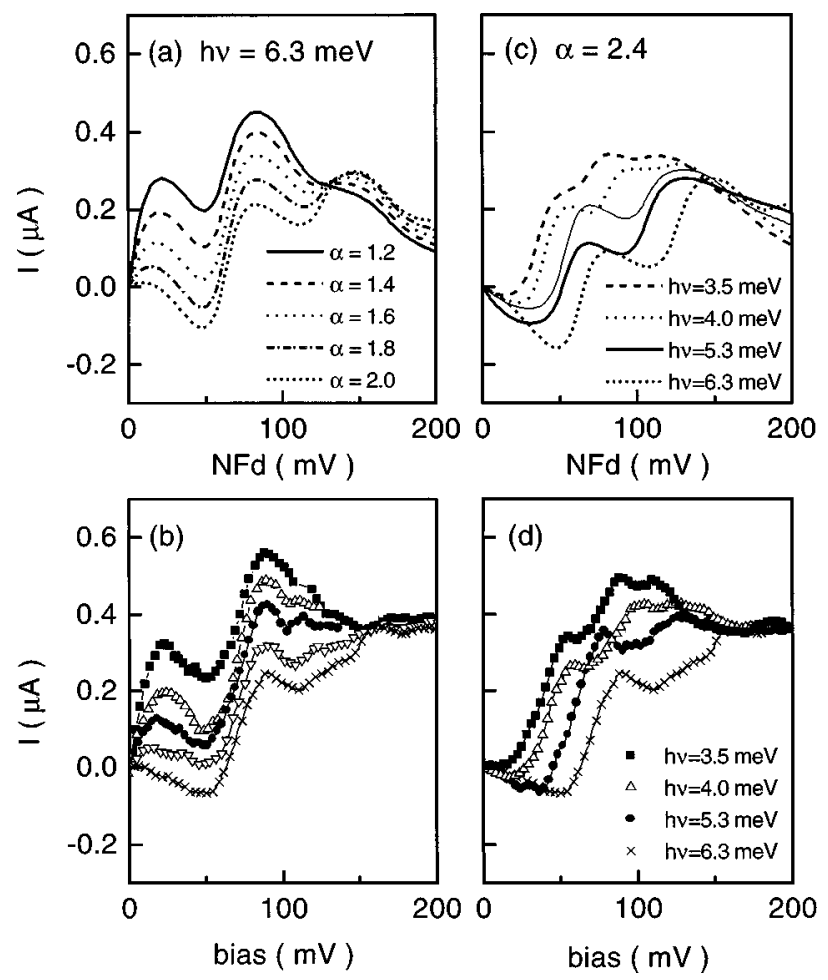

FIG. 12. Current-voltage characteristics under irradiation. (a) Theoretical results for $h \nu=6.3 \mathrm{meV}$ and different field strength $e F_{\mathrm{ac}} d=\alpha h \nu$ of the irradiation. (b) Experimental results for $h \nu=6.3$ $\mathrm{meV}$ and different laser intensities increasing from the top to the bottom. The actual values $F_{\text {ac }}$ inside the sample are not accessible. (c) Theoretical results for $\alpha=2.4$ and different photon energies. The thin line depicts $h \nu=5.3 \mathrm{meV}$ and $\alpha=2.1$. (d) Experimental results for different photon energies. The laser intensity was tuned to give maximum negative conductance.

tunneling channel is suppressed close to the zero of $J_{0}(\alpha)$ in Eq. (14), i.e., $\alpha \approx 2.4$, as used in the calculation [Fig. 12(c)]. Both the theoretical and experimental results show that absolute negative conductance persists in a wide range of frequencies but becomes less pronounced with decreasing photon energy. In the calculation absolute negative conductance vanishes for $h \nu<1.8 \mathrm{meV}$, which is approximately equal to $h \nu \lesssim e F_{\text {low }} d$. The latter relation has been verified by calculations for different samples as well. For $h \nu=5.3 \mathrm{meV}$ a smaller value of $\alpha=2.1$ (thin line) agrees better with the experimental data [in the same sense the value $\alpha=2.0$ agrees better for $h \nu=6.3 \mathrm{meV}$; compare Fig. 12(b)]. This may be explained as follows: If strong NDC is present in doped superlattices the homogeneous field distribution becomes unstable and either self-sustained oscillations or stable field domains form. ${ }^{35}$ Then the $I-V$ deviates from the relation for homogeneous field distribution, where $U=N F d$, and typically shows less pronounced NDC. Therefore maximal negative conductance is observed at a laser field corresponding to a value of $\alpha<2.4$, where the NDC is weaker and the field distribution is still homogeneous. The presence of an inhomogeneous field distribution could explain the deviations between theory and experiment for $U>150 \mathrm{mV}$ as well.

\section{DISCUSSION}

We have investigated the electrical transport in weakly coupled doped superlattices, where the transport is given by sequential tunneling. Our calculations give negative differential conductance for all doping densities and temperatures at sufficiently large electric fields. This will give rise to instabilities leading to domain formation ${ }^{36,37}$ or self-sustained current oscillations. ${ }^{4}$ Within the full transport model using Eq. (2), these effects are discussed in Ref. 7.

For high doping $N_{D} \gtrsim 10^{11} / \mathrm{cm}^{2}$ or high temperatures $k_{B} T \gtrsim\left|E_{\min }\right|$, the electrons mainly occupy free quasiparticle states. Then the general behavior can be understood within a phenomenological model using a constant self-energy $-i \Gamma / 2$. The current exhibits a maximum at $e F d \approx \Gamma$ which can be used to investigate scattering processes. For doped samples, impurity scattering is an important scattering process which we considered here. The inclusion of further scattering processes like interface roughness scattering, electronelectron scattering, or phonon scattering will increase $\Gamma$ and therefore the position of the first peak.

For low-doped samples $N_{D} \ll 10^{11} / \mathrm{cm}^{2}$ and low temperatures $k_{B} T \ll\left|E_{\min }\right|$ the presence of impurity bands influences significantly the low-field transport. Then a second maximum at $e F_{\text {high }} d \approx\left|E_{\min }\right|$ occurs. This maximum provides a possibility to obtain information about the position of the impurity band. This position depends strongly on the screening as can be seen by comparison of the calculations within the RPA and TF approximation. Therefore such experiments could serve as a test on various models for screening. For the sample considered here $E_{\min }$ calculated within the freeparticle RPA is too low compared with the experimental onset of domain formation. In contrast, the result using TF screening gives excellent agreement with the experimental data. This indicates that screening within the free-particle RPA is too strong for low-doped samples.

Furthermore it would be interesting to see if effects due to spin splitting of the impurity band are visible in experiments. These experiments can be both carried out in doped superlattices as well as in resonant tunneling between neighboring two-dimensional electron gases in the spirit of Refs. 10 and 11. The latter has the advantage that problems due to domain formation in the region of negative differential conductivity are absent. An important aspect in such experiments is the problem of electron heating as the temperature $T_{e}$ refers to the temperature of the electronic distribution. In order to avoid heating, structures with thick barriers should be used where the Joule heating becomes small.

For external irradiation we have demonstrated both experimentally and theoretically that absolute negative differential conductance persists in a wide range of frequencies $h \nu \gtrsim e F_{\text {low }} d$. The calculated current-voltage characteristics are in excellent quantitative agreement with the experimental data using a microscopically calculated $I^{\mathrm{dc}}(e F d)$ combined with the Tucker formula (14). Recently, the same model was applied to photon-assisted tunneling in a different sample, ${ }^{38}$ and quantitative agreement achieved as well. This shows that the simple Tucker formula allows for a quantitative description of photon-assisted transport in weakly coupled superlattices.

\section{ACKNOWLEDGMENTS}

We want to thank Ben $\mathrm{Hu}$ for stimulating discussions. A.W. and S.Z. acknowledge financial support by the 
Deutsche Forschungsgemeinschaft. Work performed at the Center for Terahertz Science and Technology was supported by the Office of Naval Research, the U.S. Army Research Office, and the National Science Foundation.

\section{APPENDIX A: CALCULATION OF THE TRANSITION ELEMENTS}

In a superlattice structure the coupling between neighboring wells $T_{1}$ is related to the dispersion relation $E(q)$ of the miniband (see Ref. 7) via

$$
T_{1}=\frac{d}{2 \pi} \int_{-\pi / d}^{\pi / d} d q E(q) e^{i q d}
$$

For a next-neighbor tight-binding model we have $E(q)=-(\Delta / 2) \cos (q d)$, and $T_{1}$ is equal to a fourth of the miniband width $\Delta$. Here we calculate $E(q)$ for a given superlattice via the Kronig-Penney model for the respective sample parameters. Furthermore we determine the Wannierfunctions $\Psi(z-j d)$ localized in well $j$ from the Blochfunctions $\phi_{q}(z)$, where we choose the phase of the Bloch functions such that the Wannier functions are maximally localized. ${ }^{24}$ These Wannier functions are used for the calculation of the matrix elements for scattering.

\section{APPENDIX B: CALCULATION OF THE SELF-ENERGIES}

We assume that the electron density in the conduction band is provided by doping of the superlattice. Thus scattering at ionized impurities is an important scattering process. In addition, there may be interface roughness scattering, phonon scattering, or electron-electron scattering, which we will neglect in the following. For weakly coupled superlattices, the dominating scattering process occurs within the wells, which are assumed to be identical. Thus the well index $j$ can be omitted. Scattering at the ionized impurities is described by the Hamiltonian

$$
\hat{H}^{\mathrm{scatt}}=\frac{1}{A} \sum_{\mathbf{k}, \mathbf{p}, \alpha} V_{\alpha}(\mathbf{p}) a^{\dagger}(\mathbf{k}+\mathbf{p}) a(\mathbf{k}),
$$

where the subscript $\alpha$ denotes the impurity located at the position $\left(\mathbf{r}_{\alpha}, z_{\alpha}\right)$. The matrix element is calculated with the Wannier functions, yielding

$$
\begin{aligned}
V_{\alpha}(\mathbf{p})= & \int d^{2} r d z e^{-i \mathbf{p} \cdot \mathbf{r}} \Psi^{*}(z) \Psi(z) \\
& \times \frac{-e^{2}}{4 \pi \epsilon_{s} \epsilon_{0} \sqrt{\left|\mathbf{r}-\mathbf{r}_{\alpha}\right|^{2}+\left(z-z_{\alpha}\right)^{2}}} \\
= & \frac{-e^{2}}{2 \epsilon_{s} \epsilon_{0} p} \int d z \Psi^{*}(z) \Psi(z) e^{-p\left|z-z_{\alpha}\right|} e^{-i \mathbf{p} \cdot \mathbf{r}_{\alpha}} .
\end{aligned}
$$

Using the bare Coulomb interaction $V_{\alpha}(\mathbf{p})$, the relevant integrals in the self-energies are divergent. Thus screening is essential for the calculation. We treat screening within the random-phase approximation (RPA) of the free-electron gas as well as within an effective Thomas-Fermi approximation (TF) (see Appendix C). With the screened impurity potential $V_{\alpha}^{\mathrm{sc}}(\mathbf{p})=V_{\alpha}(\mathbf{p}) / \epsilon(\mathbf{p})$ the self-energy is calculated within the self-consistent single-site approximation (shown diagrammatically in Fig. 1) as in Ref. 13. Then the self-energy contribution from the impurity $\alpha$ is given by

$$
\begin{aligned}
\sum_{\alpha}(\mathbf{k}, E)= & \frac{1}{A^{2}} \sum_{\mathbf{k}_{1}} V_{\alpha}^{\mathrm{sc}}\left(\mathbf{k}-\mathbf{k}_{1}\right) G\left(\mathbf{k}_{1}, E\right) V_{\alpha}^{\mathrm{sc}}\left(\mathbf{k}_{1}-\mathbf{k}\right) \\
& +\frac{1}{A^{3}} \sum_{\mathbf{k}_{1}, \mathbf{k}_{2}} V_{\alpha}^{\mathrm{sc}}\left(\mathbf{k}-\mathbf{k}_{1}\right) G\left(\mathbf{k}_{1}, E\right) V_{\alpha}^{\mathrm{sc}}\left(\mathbf{k}_{1}-\mathbf{k}_{2}\right) \\
& \times G\left(\mathbf{k}_{2}, E\right) V_{\alpha}^{\mathrm{sc}}\left(\mathbf{k}_{2}-\mathbf{k}\right)+\cdots,
\end{aligned}
$$

where $G(\mathbf{k}, E)=\left[E-E_{k}-\Sigma^{\text {ret }}(\mathbf{k}, E)\right]^{-1}$ is the full retarded Green function, and

$$
\Sigma^{\mathrm{ret}}(\mathbf{k}, E)=\sum_{\alpha} \Sigma_{\alpha}(\mathbf{k}, E)
$$

is the sum over all contributions. In case of $\delta$ doping the impurity potentials (B2) from different impurities located in the same well differ only by a phase factor, and the sum over $\alpha$ can be replaced by a multiplication with the number of impurities per layer $N_{D} A$. Equation (B3) can be transformed to the self-consistent equation (see, e.g., Ref. 13)

$$
\begin{aligned}
K_{\alpha}\left(\mathbf{k}_{1}, \mathbf{k}, E\right)= & V_{\alpha}^{\mathrm{sc}}\left(\mathbf{k}_{1}-\mathbf{k}\right)+\frac{1}{A} \sum_{\mathbf{k}_{2}} V_{\alpha}^{\mathrm{sc}}\left(\mathbf{k}_{1}-\mathbf{k}_{2}\right) \\
& \times G\left(\mathbf{k}_{2}, E\right) K_{\alpha}\left(\mathbf{k}_{2}, \mathbf{k}, E\right),
\end{aligned}
$$

which we solve numerically for a given self-energy function $\Sigma^{\text {ret }}(\mathbf{k}, E)$ entering $G\left(\mathbf{k}_{2}, E\right)$. We parametrize $\mathbf{k}_{1}, \mathbf{k}$ by $E_{k_{1}}, E_{k}$, and $\phi=\angle\left(\mathbf{k}_{1}, \mathbf{k}\right)$, and discretize the resulting equation. This gives a set of linear equations for the components of $K\left(E_{k_{1}}, \phi\right)$ which is solved by matrix inversion. Then the self-energy reads

$$
\Sigma_{\alpha}(\mathbf{k}, E)=\frac{1}{A^{2}} \sum_{\mathbf{k}_{1}} V_{\alpha}^{\mathrm{sc}}\left(\mathbf{k}_{1}-\mathbf{k}\right) G\left(\mathbf{k}_{1}, E\right) K_{\alpha}\left(\mathbf{k}_{1}, \mathbf{k}, E\right) .
$$

Equations (B4), (B5), and (B6) have to be solved selfconsistently, thus determining the self-energy $\Sigma^{\mathrm{ret}}(\mathbf{k}, E)$.

Our single-site-approximations neglects all contributions from crossed diagrams (leading to weak-localization effects, as considered in Ref. 39) as well as the spin-resolved electron-electron interaction leading to the splitting of the impurity bands (the Mott transition; see, e.g., Ref. 40). The latter may become important for very low densities when the impurity bands are narrow. Within this approximation for $\sum^{\text {ret }}(\mathbf{k}, E)$, integral (4) is a well-defined quantity, as $\operatorname{Im}\left\{\Sigma^{\text {ret }}(\mathbf{k}, E)\right\}=0$ (and thus $A(\mathbf{k}, E)=0$ ) for $E<E_{\min }$.

Finally note that no impurity bands are found within the self-consistent Born approximation

$$
\Sigma_{\alpha}(\mathbf{k}, E)=\frac{1}{A^{2}} \sum_{\mathbf{k}_{1}} V_{\alpha}^{\mathrm{sc}}\left(\mathbf{k}-\mathbf{k}_{1}\right) G\left(\mathbf{k}_{1}, E\right) V_{\alpha}^{\mathrm{sc}}\left(\mathbf{k}_{1}-\mathbf{k}\right),
$$

which is just the first diagram from Fig. 1. 


\section{APPENDIX C: SCREENING}

In order to consider screening we have to include the electron-electron interaction given by the Hamiltonian

$$
\hat{H}^{e e}=\frac{1}{2 A} \sum_{\mathbf{k}, \mathbf{k}^{\prime}, \mathbf{p}} W(\mathbf{p}) a^{\dagger}(\mathbf{k}+\mathbf{p}) a^{\dagger}\left(\mathbf{k}^{\prime}-\mathbf{p}\right) a\left(\mathbf{k}^{\prime}\right) a(\mathbf{k}),
$$

where the matrix element is calculated via

$$
\begin{aligned}
W(\mathbf{p})= & \frac{e^{2}}{2 \epsilon_{s} \epsilon_{0} p} \int d z_{1} \int d z_{2} \Psi^{*}\left(z_{1}\right) \Psi^{*}\left(z_{2}\right) \\
& \times \Psi\left(z_{2}\right) \Psi\left(z_{1}\right) e^{-p\left|z_{1}-z_{2}\right|} .
\end{aligned}
$$

Within the RPA, the screening of the impurity potentials is described by ${ }^{25}$

$$
V_{\alpha}^{\mathrm{RPA}}(\mathbf{p})=\frac{V_{\alpha}(\mathbf{p})}{1-\Pi^{0}(\mathbf{p}, \omega=0) W(\mathbf{p})} .
$$

For a free-electron gas the two-dimensional vacuum polarizability $\Pi^{0}(\mathbf{p}, \omega=0)$ for $T=0$ is given by ${ }^{41}$

$$
\Pi^{0}(\mathbf{p}, \omega=0)=-\rho_{0}\left[1-\Theta\left(p-2 k_{F}\right)\left(1-4 \frac{k_{F}^{2}}{p^{2}}\right)^{1 / 2}\right],
$$

where $k_{F}=\left(2 \pi N_{D}\right)^{1 / 2}$ is the Fermi wave vector.

Actually, the electronic states are affected by the impurity scattering, which may change the density of states dramatically as can be seen from Fig. 2. Now the polarizability $\Pi(p=0)$ is related to the actual density of states at the chemical potential which is significantly lower than $\rho_{0}$. Calculations within the Born approximation show that the $p$ dependence of the polarizability becomes weaker, and that $\Pi(0)$ decreases with increasing scattering. ${ }^{42,43}$ In order to accommodate these trends we make the replacement $\Pi^{0}(k) \rightarrow \Pi^{*}(k)=-\rho\left(E^{F}\right)$, given by the calculated density of states of Fig. 2 and the chemical potential at $T=0$. Then we obtain the screened impurity interaction

$$
V_{\alpha}^{\mathrm{TF}}(\mathbf{p})=\frac{V_{\alpha}(\mathbf{p})}{1+\rho\left(E^{F}\right) W(\mathbf{p})} .
$$

This is equivalent to the Thomas-Fermi approximation for the screening. The same type of screening has been considered in Ref. 13 as well. Of course both ways of including screening are approximations. In a full calculation the scattering has to be treated self-consistently in the calculation of the polarizability. Such a calculation was performed in Ref. 44 for a quantum wire within the restriction of a deltapotential for impurity scattering.

Equation (C3) only considers screening within the same well. The extension to screening by electrons from neighboring wells is given in Sec. 6 of Ref. 7. The results are almost indistinguishable for the samples with thick barrier width considered in Secs. IV and V (see also Ref. 12 for the screened matrix elements). Screening by electrons from neighboring wells becomes more important for a smaller barrier width as used in Secs. VI and VII, where the formalism from Ref. 7 was used with the polarization (C4) for the RPA and $\Pi^{0}(k)=-\rho\left(E^{F}\right)$ for the TF case. The temperature dependence of the screening is neglected in all calculations.
*Present address: Klarastr. 5a, 80636 München, Germany.

${ }^{1}$ L. Esaki and L. L. Chang, Phys. Rev. Lett. 33, 495 (1974).

${ }^{2}$ F. Capasso, K. Mohammed, and A. Y. Cho, Appl. Phys. Lett. 48, 478 (1986).

${ }^{3}$ S. H. Kwok, H. T. Grahn, M. Ramsteiner, K. Ploog, F. Prengel, A. Wacker, E. Schöll, S. Murugkar, and R. Merlin, Phys. Rev. B 51, 9943 (1995).

${ }^{4}$ J. Kastrup, R. Hey, K. H. Ploog, H. T. Grahn, L. L. Bonilla, M. Kindelan, M. Moscoso, A. Wacker, and J. Galán, Phys. Rev. B 55, 2476 (1997).

${ }^{5}$ L. Esaki and R. Tsu, IBM J. Res. Dev. 14, 61 (1970).

${ }^{6}$ B. Laikhtman and D. Miller, Phys. Rev. B 48, 5395 (1993).

${ }^{7}$ A. Wacker, in Theory of Transport Properties of Semiconductor Nanostructures, edited by E. Schöll (Chapman and Hall, London, in press).

${ }^{8}$ R. F. Kazarinov and R. A. Suris, Fiz. Tekh. Poluprovodn. 6, 148 (1972) [Sov. Phys. Semicond. 6, 120 (1972)].

${ }^{9}$ L. Zheng and A. H. MacDonald, Phys. Rev. B 47, 10619 (1993).

${ }^{10}$ S. Q. Murphy, J. P. Eisenstein, L. N. Pfeiffer, and K. W. West, Phys. Rev. B 52, 14825 (1995).

${ }^{11}$ N. Turner, J. T. Nicholls, E. H. Linfield, K. M. Brown, G. A. C. Jones, and D. A. Ritchie, Phys. Rev. B 54, 10614 (1996).

${ }^{12}$ A. Wacker and A.-P. Jauho, Phys. Scr. T69, 321 (1997).

${ }^{13}$ A. Gold, J. Serre, and A. Ghazali, Phys. Rev. B 37, 4589 (1988).

${ }^{14}$ J. Serre, A. Ghazali, and A. Gold, Phys. Rev. B 39, 8499 (1989).

${ }^{15}$ P. S. S. Guimaraes, B. J. Keay, J. P. Kaminski, S. J. Allen, P. F. Hopkins, A. C. Gossard, L. T. Florez, and J. P. Harbison, Phys. Rev. Lett. 70, 3792 (1993).
${ }^{16}$ B. J. Keay, S. J. Allen, J. Galán, J. P. Kaminski, K. L. Champman, A. C. Gossard, U. Bhattacharya, and M. J. M. Rodwell, Phys. Rev. Lett. 75, 4098 (1995).

${ }^{17}$ J. Inarrea and G. Platero, Europhys. Lett. 34, 43 (1996).

${ }^{18}$ B. J. Keay, S. Zeuner, S. J. Allen, K. D. Maranowski, A. C. Gossard, U. Bhattacharya, and M. J. M. Rodwell, Phys. Rev. Lett. 75, 4102 (1995).

${ }^{19}$ S. Zeuner, B. J. Keay, S. J. Allen, K. D. Maranowski, A. C. Gossard, U. Bhattacharya, and M. J. W. Rodwell, Phys. Rev. B 53, 1717 (1996).

${ }^{20}$ G. Platero and R. Aguado, Appl. Phys. Lett. 70, 3546 (1997).

${ }^{21}$ P. K. Tien and J. P. Gordon, Phys. Rev. 129, 647 (1963).

${ }^{22}$ J. R. Tucker and M. J. Feldman, Rev. Mod. Phys. 57, 1055 (1985).

${ }^{23}$ M. Wagner, Phys. Rev. Lett. 76, 4010 (1996).

${ }^{24}$ W. Kohn, Phys. Rev. 115, 809 (1959).

${ }^{25}$ G. D. Mahan, Many-Particle Physics (Plenum, New York, 1990).

${ }^{26}$ D. Miller and B. Laikhtman, Phys. Rev. B 50, 18426 (1994).

${ }^{27}$ G. Brozak, M. Helm, F. DeRosa, C. H. Perry, M. Koza, R. Bhat, and S. J. Allen, Phys. Rev. Lett. 64, 3163 (1990).

${ }^{28}$ P. A. Lebwohl and R. Tsu, J. Appl. Phys. 41, 2664 (1970).

${ }^{29}$ A. Y. Shik, Fiz. Tekh. Poluprovodn. 8, 1841 (1974) [Sov. Phys. Semicond. 8, 1195 (1975)].

${ }^{30}$ Properties of Aluminium Gallium Arsenide, edited by S. Adachi (INSPEC, London, 1993).

${ }^{31}$ P. Helgesen and T. G. Finstad, in Proceedings of the 14th Nordic Semiconductor Meeting, edited by O. Hansen (University of Århus, Århus, Denmark, 1990), p. 323. 
${ }^{32}$ P. Helgesen, T. G. Finstad, and K. Johannessen, J. Appl. Phys. 69, 2689 (1991).

${ }^{33}$ W. Hilber, M. Helm, K. Alavi, and R. N. Pathak, Appl. Phys. Lett. 69, 2528 (1996).

${ }^{34}$ A. A. Ignatov, E. Schomburg, J. Grenzer, K. F. Renk, and E. P. Dodin, Z. Phys. B 98, 187 (1995).

${ }^{35}$ A. Wacker, M. Moscoso, M. Kindelan, and L. L. Bonilla, Phys. Rev. B 55, 2466 (1997).

${ }^{36}$ F. Prengel, A. Wacker, and E. Schöll, Phys. Rev. B 50, 1705 (1994); 52, 11518 (1995).

${ }^{37}$ L. L. Bonilla, J. Galán, J. A. Cuesta, F. C. Martínez, and J. M.
Molera, Phys. Rev. B 50, 8644 (1994).

${ }^{38}$ A. Wacker and A.-P. Jauho, Phys. Status Solidi B (to be published).

${ }^{39}$ W. Szott, C. Jedrzejek, and W. P. Kirk, Phys. Rev. B 45, 3565 (1992).

${ }^{40}$ B. I. Shklovskii and A. L. Efros, Electronic Properties of Doped Semiconductors (Springer, Berlin, 1984).

${ }^{41}$ F. Stern, Phys. Rev. Lett. 18, 546 (1967).

${ }^{42}$ T. Ando, J. Phys. Soc. Jpn. 51, 3215 (1982).

${ }^{43}$ S. Das Sarma, Phys. Rev. Lett. 50, 211 (1983).

${ }^{44}$ B. Y. Hu and S. Das Sarma, Phys. Rev. B 48, 5469 (1993). 